\title{
Análisis del cumplimiento de revelación de propiedades, planta y equipo para empresas que listan en la bolsa de valores de Perú
}

\author{
Julio Hernández-Pajares ${ }^{1}$ \\ Ivette Núñez Laguna²
}

Recibido: 30 de septiembre de 2019

Aprobado: 04 de diciembre de 2019

Hernández-Pajares, J., y Núñez, I. (2020). Análisis del cumplimiento de revelación de propiedades, planta y equipo para empresas que listan en la bolsa de valores de Perú. Revista Activos, 18(1), 205-233. DoI: https://doi. org/10.15332/25005278/6163

\section{Clasificación JEL: M41}

\section{Resumen}

La adopción de las Normas Internacionales de Información Financiera (NIIF) buscan mejorar la calidad, transparencia y comparabilidad de la información financiera en las empresas. Por ello, la presente investigación tiene como objetivo realizar un estudio sobre la naturaleza de los criterios de revelación de las propiedades, planta y equipo, según la NIC 16, y determinar

1 Doctor en Contabilidad y Finanzas. Facultad de Ciencias Económicas y Empresariales, Universidad de Piura-Campus Lima. Correo electrónico: julio.hernandez@udep.edu.pe.

ORCID: https://orcid.org/0000-0002-7481-2912

2 Contadora Pública Facultad de Ciencias Económicas y Empresariales, Universidad de Piura-Campus Lima. Correo electrónico: ivette.nunez@udep.edu.pe. ORCID: http://orcid.org/0000-0001-8524-0588 
la influencia de factores en su nivel de cumplimiento. Para este propósito, se realizó un análisis de contenido de los informes anuales de las compañías que se encuentran listadas en la bolsa de valores de Perú para los periodos anuales 2016, 2017 y 2018. Los resultados indican que, en su mayoría, las empresas revelan su base de medición, métodos de depreciación y vida útil de los activos. En este sentido, el sector empresarial, la inversión en PPE, antigüedad y firma de auditoría resultan factores determinantes en el nivel de dicha revelación.

Palabras clave: información financiera; NIIF; propiedades, planta y equipo; revelación financiera; firma de auditoría.

\title{
Analysis of the Compliance with the Disclosure of Property, Plant, and Equipment of Companies listed in the Peruvian Stock Exchange
}

\begin{abstract}
The adoption of International Financial Reporting Standards (IFRs) seeks to improve the quality, transparency, and comparability of financial reporting in companies. Therefore, the purpose of this research is to analyse the nature of the disclosure criteria for property, plant, and equipment in accordance with IAS 16, and to determine the influence of several factors on their level of compliance. For this purpose, the content of the annual reports of the companies listed on the Peruvian Stock Exchange for the annual periods of 2016, 2017 and 2018 was carried out. The results indicate that, for the most part, companies disclose their measurement basis, their depreciation methods and the useful life of their assets. In this sense, the business sector, investment in PPE, seniority, and audit firms are determining factors in the level of such disclosure.
\end{abstract}

Keywords: financial information; IFRS; property, plant, and equipment; financial disclosure; audit firms. 


\section{Introducción}

Es muy importante para las organizaciones e inversionistas el crecimiento de las empresas, es decir, determinar de qué manera pueden expandir su negocio. Este puede generarse a través de un crecimiento orgánico o inorgánico. Por un lado, el inorgánico se genera con la adquisición o fusión de empresas y podría aumentar rápidamente el tamaño y los ingresos. Por otro lado, el orgánico es una forma de expandir el negocio aumentando la producción y mejorando las ventas de forma interna, además de representar un compromiso sólido y a largo plazo. La adquisición de activos fijos es una forma de crecimiento orgánico en la organización y es por ello su importancia tanto para la administración como para los stakeholders, necesitan presentar y recibir información que refleje la realidad económica de la empresa (Johnston, 2014).

Dentro de este grupo de activos fijos no financieros y no corrientes, se encuentran las propiedades, planta y equipo (PPE), las cuales son utilizadas en la producción, suministro de bienes y servicios, para ser arrendados a terceros o para fines administrativos y se esperan utilizar por más de un periodo, de no cumplir con las características deberá ser registrado como gasto del ejercicio.

Las organizaciones deben reflejar en sus estados financieros (EEFF) la adquisición o producción de PPE. Con la adopción de la Normativa Internacional de Información Financiera (NIIF o IRFS por sus siglas en inglés) en el mundo, se busca mejorar la calidad de la información financiera y la eficiencia de los mercados públicos permitiendo una información más armonizada (Choi y Meek, 2008; Horton et ál., 2013). Debe considerarse que el proceso de transición de las normas locales a las NIIF, en los países obligados a ello, tuvo dificultades debido a la falta de una efectiva regulación y experiencia profesional (Callao et ál., 2007; Cascino y Gassen, 2015; Daske et ál., 2013).

En Latinoamérica el proceso de adopción de NIIF y armonización no fue similar para todos los países. En algunos casos la aplicación requirió 
del esfuerzo para minimizar las discrepancias entre normas locales y NIIF. En otros casos, no todas las empresas adoptaron completamente la normativa, solo lo hicieron las empresas reguladas por entidades del mercado de valores de cada país (Cano 2010; Jara et ál., 2010; Silva y Garrido, 2006).

Respecto al caso peruano, antes de la transición a NIIF, los EEFF se preparaban con base en principios de contabilidad generalmente aceptados. Para la adopción de NIIF se emitieron normas como el Plan Contable Empresarial (PCGE) basado en las NIIF por el Consejo Normativo de Contabilidad con aplicación a partir del año 2011; la Ley 29720 y su reglamento que en su artículo $5 .^{\circ}$ obliga a las empresas a presentar sus EefF bajo NIIF y la Resolución de la Superintendencia del Mercado de Valores (SMV) 011-2012. SMV/01, que obligaba a las empresas además a presentar los EEFF bajo NIIF auditados a partir del año 2012.

Las investigaciones en empresas peruanas sobre información financiera bajo NIIF han tenido distintos enfoques. Se encuentran estudios sobre la naturaleza de los criterios de medición y revelación, así como de las limitaciones en la adopción de la NIIF debido a la influencia de la regulación contable anterior y la fiscal (Díaz-Becerra; 2010; Hernández et ál., 2017; Molina-Llopis et ál., 2014; Mongrut y Winkelried, 2019; Salas et ál., 2017). Cabe señalar que otros estudios encuentran que el nivel cumplimiento de la NIIF en Perú se ha visto influenciado por el tamaño, sector y firma de auditora de las empresas cotizadas (Pocomucha y Hernández-Pajares, 2019).

La Norma Internacional de Contabilidad (NIC) 16 tiene como objetivo prescribir el tratamiento contable de propiedades, planta y equipo, de forma que los usuarios de los eEfF puedan conocer la información acerca de la inversión que la entidad tiene de ellos, así como los cambios producidos en dicha inversión.

La norma entró en vigor a partir del 1. ${ }^{\circ}$ de enero de 2005. Una de las últimas modificaciones a la norma se emitió en junio de 2014 y entró en 
vigor para los periodos anuales del $1 .^{\circ}$ de enero de 2016, la cual incluye el reconocimiento de plantas productoras ${ }^{3}$, otra de estas modificaciones la emitió la NIIF 16 Arrendamientos, que elimina los párrafos 4 y 27 y modifica los párrafos 5 y 10 , que entrará en vigencia cuando se aplique la nueva normativa de arrendamientos. La NIC 16 resulta una de las normas más relevantes y de mayor aplicación para la preparación de EEFF (Aljifri y Khasharmeh, 2006; Paik, 2009).

Investigaciones como las de Cardona (2019), De Moura y Gupta (2019) y Yáñez-Andrades et ál. (2010) señalan la importancia de la adopción de NIIF por las empresas en países de economía emergente y específicamente en Latinoamérica, respecto a la transparencia, confiabilidad y comparabilidad en la presentación de información financiera a los usuarios de la información. Todas estas razones nos motivan a analizar el nivel de cumplimiento de la revelación de las PPE en los EEFF e identificar cuáles son los factores que podrían influir en el nivel de revelación exigida por la normativa contable. Para ello, se realizó un análisis de contenido de los reportes de 138 empresas peruanas listadas en bolsa, las cuales aplican NIIF completas en sus EEFF. Para el análisis relacional se realizó una regresión lineal múltiple de algunas variables (sector empresarial, tamaño de la empresa, inversión en PPE, antigüedad de la empresa, firma de auditoría y rentabilidad) seleccionadas como determinantes en el nivel de cumplimiento de divulgación requerido por la NIC 16.

En esta investigación se presentan antecedentes teóricos sobre la contabilización y presentación de los elementos de PPE según la NIC 16 y expone los factores que pueden influir en los criterios de revelación y planteamiento de hipótesis. La tercera parte explica el diseño de la investigación, la cuarta el análisis de los resultados para finalmente presentar las conclusiones.

3 Una planta productora es una planta viva que se utiliza en la elaboración o suministro de productos agrícolas. Se espera que produzca durante más de un periodo y tiene una probabilidad remota de ser vendida como productos agrícolas, excepto por ventas incidentales de raleos y podas. 


\section{Marco teórico}

Si bien el objetivo de la NIC 16 es prescribir el tratamiento contable de propiedades, planta y equipo, también expone los principales problemas que presentan su reconocimiento, como la contabilidad de sus activos, la determinación de su importe en libros ${ }^{4}$ y los cargos por depreciación ${ }^{5}$ y pérdidas por deterioro ${ }^{6}$, que deben reconocerse con relación a ellos mismos. El Consejo de Normas Internacionales de Contabilidad (IASB por sus siglas en inglés) busca que con las NIIF se maneje información de alta calidad, transparente y comparable, que permita tomar decisiones con fundamento.

Las empresas que presentan en sus EEFF los elementos de PPE, de acuerdo con la NIC 16, deben revelar información sobre los criterios de medición inicial y posterior bajo los modelos del costo e información detallada revelados en la nota de políticas contables y notas explicativas información. Si bien las empresas deben reconocer inicialmente sus PPE bajo el modelo del costo histórico ${ }^{7}$, para su valoración posterior pueden optar por elegir el modelo del costo histórico o el modelo de revaluación ${ }^{8}$ como política contable y aplicará esa política a todos los elementos que compongan una clase.

4 Importe en libros: es el importe por el que se reconoce un activo, una vez deducidas la depreciación acumulada y las pérdidas por deterioro del valor acumulado.

5 Depreciación es la distribución sistemática del importe depreciable de un activo a lo largo de su vida útil.

6 La pérdida por deterioro es el exceso del importe en libros de un activo sobre su importe recuperable.

7 Modelo del costo: costo menos depreciación y el importe acumulados de las pérdidas por deterioro.

8 Valor revaluado: valor razonable en el momento de la revaluación menos la depreciación y el importe acumulados de las pérdidas por deterioro de valor que haya sufrido. 
Tomando en cuenta la literatura existente sobre el impacto de la adopción de IFRS, distintos estudios han abordado los criterios de medición bajo la NIC 16, donde se encuentra que en su mayoría las empresas han optado por el modelo del costo histórico para valorar su PPE y optan por el método de línea recta para depreciarlos. (Ahmed et ál., 2002; Católico-Segura et ál., 2013; Díaz-Becerra et ál., 2012; Pachón, 2010; Paik, 2009; Reeh y Molina, 2013). El modelo de revaluación se presenta como una opción de medición a valor razonable que incide en la situación financiera y solvencia de las empresas, algunos autores inciden en que este modelo de valoración no ha resultado una opción generalmente aplicada para mejorar la percepción de los inversionistas y acreedores sobre la situación financiera de las empresas (Jaggi y Tsui 2001; Hu et ál., 2015; Missonier-Piera, 2007).

Las políticas contables señaladas anteriormente, se optaron en algunos casos por los incentivos tributarios para elegirla práctica (Barlev et ál. 2007; Choi et ál., 2013; Easton et ál.,1993). En el Perú, la revaluación de PPE no tiene beneficios tributarios y su aplicación implica una desventaja debido al reparo tributario de la depreciación de los mayores valores revaluados (Díaz-Becerra et ál., 2012).

En la presente investigación, el análisis de los factores que influyen en el cumplimiento de revelación de PPE se basa en las teorías de agencia y costo del propietario. Las investigaciones que estudian la teoría de la agencia señalan que la revelación sobre información financiera que presentan grandes empresas es mayor que las pequeñas o medianas, con el fin de reducir los potenciales conflictos de intereses y, por lo tanto, los costos de agencia con los accionistas y justificar su endeudamiento (Ahmed y Courtis, 1999; Giner et ál., 2003). Otros estudios señalan que la revelación sobre información financiera que presentan grandes empresas es mayor con el fin de reducir los potenciales conflictos de intereses, por lo tanto, los costos de agencia con los accionistas (Meek y Gray, 1989; García y Sánchez, 2006).

Por su parte, la teoría de costos de propiedad señala que los directivos pueden optar por divulgar menos información de sus empresas para reducir 
los costos que genera (costo económico y costo estratégico) (Birt et ál., 2006; Miihkinen, 2012).

Sobre las ideas expuestas por diversos autores seleccionamos posibles factores que pueden influir en el nivel de divulgación financiera de las empresas: sector empresarial, inversión en PPE, tamaño, antigüedad, firma de auditoría y rentabilidad, que explicaremos a continuación.

El sector empresarial y su impacto en el nivel de información revelada en los reportes anuales es estudiado por diversas investigaciones (Cooke, 1992; Santos et ál., 2014; Lang y Stice-Lawrence, 2015). El giro de negocio y la naturaleza de las actividades empresariales podría generar un mayor impacto en algunas industrias que en otras (Ferreira da Costa y Morais de Oliveira, 2015; Kang y Gray, 2011; Stadler y Nobes, 2014; Tawiah y Boolaky, 2019).

Con respecto a la divulgación de información de PPE, algunos estudios señalan que el tamaño de la empresa es un factor determinante en los niveles de información (Mutawaa y Hewaidy, 2010; Erdem et ál., 2017; Istiningrum y Za’im Muhtadi, 2018; Uyar et ál., 2016).

La antigüedad de las empresas también resulta determinante en el nivel de divulgación financiera. Aquellas con mayor experiencia en preparar información financiera tienen menores costos de preparación y deben cumplir con una mayor regulación, por lo que presentan una mejor información financiera (Alanezi y Albuloushi, 2011; Kang y Gray, 2011; Owusu-Ansah,1998).

Estudiar el impacto que la firma auditora tendría en el nivel de cumplimiento de las revelaciones es importante porque podría identificarse si existe una mayor o menor calidad o cantidad de información presentada. (DeAngelo, 1981; García y Sánchez, 2006). Las firmas más reconocidas en el mundo denominadas big four ${ }^{9}$ influyen en el detalle de los criterios de 
divulgación y en la presentación de información en cumplimiento de la normativa exigida y en las notas a los EefF (Ferreira da Costa, y Morais de Oliveira, 2015; Santos etál., 2014; Agyei-Mensah, 2019; Owusu-Ansah,1998; Hellman et ál., 2018).

Finalmente, debemos analizar si la administración de las empresas con mejor rendimiento tendría mayores incentivos para divulgar un mejor nivel de revelación de su información financiera. Uno de los argumentos de la literatura es que estarían motivados a presentar una información más transparente y de mejor calidad para ingresar a mercados públicos para incrementar sus inversiones (Lang y Lundholm, 1993). Autores como Istiningrum y Zảim Muhtadi (2018) encuentran relación entre el nivel de revelación de PPE y el rendimiento, pero en otros no se ha identificado ninguna relación (Mutawaa y Hewaidy, 2010; Uyar et ál., 2016).

Los estudios no son concluyentes respecto a la relación entre la rentabilidad y revelación financiera. En algunos casos la relación es positiva y significativa (Lang y Stice-Lawrence, 2015; Santos et ál., 2014), pero en otros casos no se encuentra dicha relación (Ahmed y Courtis, 1999; Barako et ál., 2006).

De acuerdo con lo señalado planteamos las siguientes hipótesis:

H1: El sector empresarial es determinante en el nivel de cumplimiento de revelación de información de PPE.

H2: La inversión en ppe respecto al total de activos es determinante en el nivel de cumplimiento de su revelación de información.

H3: El tamaño de las empresas es determinante en el nivel de cumplimiento de revelación de información de PPE.

H4: La antigüedad de las empresas es determinante en el nivel de cumplimiento de revelación de información de PPE. 
H5: La firma de auditoría es determinante en el nivel de cumplimiento de información de activos intangibles.

H6: La rentabilidad de las empresas es determinante en el nivel de cumplimiento de información de PPE.

\section{Metodología}

\section{Muestra}

Los reportes anuales auditados utilizados para este estudio que comprenden el dictamen de auditoría y EEFF de las empresas se han obtenido de la base de datos de la página web de la Superintendencia del Mercado de Valores (SMv). Se tomó la población total de organizaciones que listaron en bolsa en los periodos anuales de 2016, 2017 y 2018 de la cual se consideró una muestra no aleatoria de 138 empresas que presentaron información para los tres años, se excluyeron de la población a los fondos de inversiones, empresas fiduciarias y titulizadoras sin inversión significativa en PPE, así como las organizaciones en liquidación y reestructuración. La muestra considera las empresas que aplican la NIC 16 para la medición y revelación de PPE como norma obligatoria.

El criterio de clasificación sectorial fue el considerado por la Bolsa de Valores de Lima (BVL) y comprenden los sectores observados en la tabla 1. La muestra de empresas según sector presenta un mayor número para los sectores financiero y seguros que incluyen bancos, financieras, cajas de ahorro y empresas de seguros y fondos de pensiones; industrial de manufacturera de bienes y de servicios públicos en su mayor parte de generación y distribución de energía, gas y agua. La industria más representativa es la financiera, con un $25 \%$. 
Tabla 1. Muestra clasificada por sectores

\begin{tabular}{|l|c|c|}
\cline { 2 - 3 } \multicolumn{1}{c|}{} & Frecuencia & Porcentaje \\
\hline Agroindustrial & 9 & $7 \%$ \\
\hline Comercial & 5 & $4 \%$ \\
\hline Construcción/inmobiliario & 8 & $6 \%$ \\
\hline Financiero/seguros & 34 & $25 \%$ \\
\hline Industria & 33 & $24 \%$ \\
\hline Minería/petróleo & 17 & $12 \%$ \\
\hline Servicios & 10 & $7 \%$ \\
\hline Servicios públicos & 22 & $16 \%$ \\
\hline Total & $\mathbf{1 3 8}$ & $\mathbf{1 0 0} \%$ \\
\hline
\end{tabular}

Fuente: elaboración propia.

\section{Diseño metodológico y variables}

Esta investigación es de naturaleza cuantitativa, longitudinal y descriptiva del nivel de cumplimento de revelación de PPE respecto a las variables de sector empresarial, inversión en PPE, tamaño y antigüedad de la empresa, firma de auditoría y rentabilidad. Se analizan los reportes anuales auditados por el periodo 2016-2018, desde la entrada en vigor de las últimas modificaciones a la NIC el 2016. Para las técnicas de análisis estadístico descriptivo se utilizó el software spss26. Asimismo, se realizó un análisis de regresión lineal múltiple de panel de datos, en aplicación del software STATA, para evaluar la influencia significativa de las variables independientes en el nivel de cumplimiento de revelación aplicado en estudios similares (Cardona, 2018).

La variable dependiente es el nivel de cumplimiento de revelación; su medición se obtuvo del análisis de contenido de las notas a los EEFF de los 
reportes que comprende la nota de políticas contables y detalle de la partida de PPE (Salas et ál., 2017). La medición de la variable dependiente de nivel de cumplimiento de los criterios de revelación en las notas consideró primero una variable dicotómica, de 0 si no cumple y 1 si cumple cada uno de los siguientes criterios de revelación del párrafo 73 de la NIC 16 :

- Las bases de medición utilizadas.

- Los métodos de depreciación utilizados.

- Las vidas útiles o las tasas de depreciación utilizadas.

- Una conciliación entre los valores en libros al principio y al final del periodo, mostrando adiciones, deterioro, revaluaciones, depreciación y otros cambios.

- Información sobre las partidas de propiedades, planta y equipo que hayan sufrido pérdidas por deterioro.

- Las propiedades, planta y equipos restringidos en su titularidad como garantía de cumplimiento de obligaciones.

- El importe de los desembolsos reconocidos, en los casos de elementos de propiedades, planta y equipo en curso de construcción.

- Información sobre la distribución de la depreciación en las cuentas de resultados.

- El importe de los compromisos de adquisición de propiedades, planta y equipo.

Posteriormente, se determinó un índice de medición de cumplimiento, como el promedio de incidencias de criterios de revelación cumplidos, señalados anteriormente (Hodgdon et ál., 2009; Samaha et ál., 2016). 
En relación con las variables independientes, el sector empresarial tuvo dos medidas. Para el análisis descriptivo se consideró la clasificación de la BVL (ver tabla 1). Para la regresión lineal múltiple se consideró una variable categórica que clasifica a los sectores en un primero grupo con valor 1, que comprenden las empresas con mayor inversión y uso de PPE como industrial manufacturera, agroindustrial, minera y petróleo y de servicios públicos de energía agua y gas y el segundo que comprende el resto de sectores de servicios, financiero y comercial, con valor 0 (Alanezi et ál., 2016; Cascino y Gassen, 2015).

Para la variable tamaño de la empresa, se consideró el valor total de los activos del año de revelación, y para la inversión en PPE se aplicó la proporción de su valor respecto al total de activos de cada año estudiado (Cooke, 1992; Ahmed y Courtis, 1999). Respecto a la antigüedad de las empresas, esta variable comprende el número de años desde su constitución hasta el periodo de medición (Alanezi y Albuloushi, 2011; Mutawaa y Hewaidy, 2010).

Para la variable firma de auditoría, se identificó la firma encargada de emitir opinión en el dictamen de cada año, y para medir su tamaño se clasificaron en dos grupos. El primero, que comprende las 5 firmas más grandes según su ubicación en el escalafón mundial (Crowe Howart, 2017), por su volumen de ventas, y denominaremos "grandes" (PWC, E\&Y, Deloitte, KPMG у вDO), y las firmas que no pertenecen al grupo anterior clasificadas como "otras" (Alanezi et ál., 2016; Hodgdon et ál., 2009; Santos et ál., 2014).

Finalmente, para la variable rentabilidad se aplicó el indicador de rentabilidad sobre activos (ROA) del periodo anterior al de la medición, obtenido de la utilidad neta del periodo respecto al total de activos de los EEFF (Cardona, 2018; Uyar et ál., 2016).

La tabla 2 muestra las mediciones de las variables independientes que se consideran para el estudio descriptivo y regresión lineal múltiple. 
Tabla 2. Medición de variables independientes

\begin{tabular}{|l|l|}
\hline Sector & $\begin{array}{l}\text { 1 para las empresas del grupo "industriales } \\
\text { y energía" y 0 para el grupo de "servicios y } \\
\text { financieras" }\end{array}$ \\
\hline Inversión de PPE & $\begin{array}{l}\text { Valor de PPE/valor total de activos para el } \\
\text { periodo }\end{array}$ \\
\hline Tamaño de empresa & Ln del valor total de activos del periodo \\
\hline Antigüedad & $\begin{array}{l}\text { Diferencia de años entre el de la constitución } \\
\text { de la empresa y periodo de medición }\end{array}$ \\
\hline Firma de auditoría & $\begin{array}{l}1 \text { si pertenece al grupo de "grandes" y 0 si } \\
\text { pertenece al grupo de "otras" del periodo }\end{array}$ \\
\hline Rentabilidad del año anterior & $\begin{array}{l}\text { Utilidad neta/valor total de activos del periodo } \\
\text { anterior de medición }\end{array}$ \\
\hline
\end{tabular}

Fuente: elaboración propia.

El modelo de regresión que considera las variables de la tabla 2 se explica a continuación:

$$
\begin{gathered}
\text { Índice de revelación }=\beta 0+\beta 1 \text { (sector })+\beta 2 \text { (inversión de PPE }) \\
+\beta 3(\text { Ln Tamaño })+\beta 4(\text { antigüedad })+\beta 5 \text { (firma de auditoría }) \\
+\beta 6(\text { rentabilidad })+\varepsilon
\end{gathered}
$$

\section{Análisis de resultados}

\section{Análisis descriptivo de criterios de medición}

El análisis del cumplimiento de revelación de la NIC 16 promedio para los tres años, como se observa en la figura 1, muestra que los criterios que presentan un mayor nivel revelación son las bases medición para las PPE, 
métodos y vidas útiles consideradas para la depreciación, así como las conciliaciones de saldos iniciales y finales del costo y depreciación acumulada de las PPE, en una media de incidencia mayor al $95 \%$.

Figura 1. Nivel de cumplimiento promedio de criterios de revelación

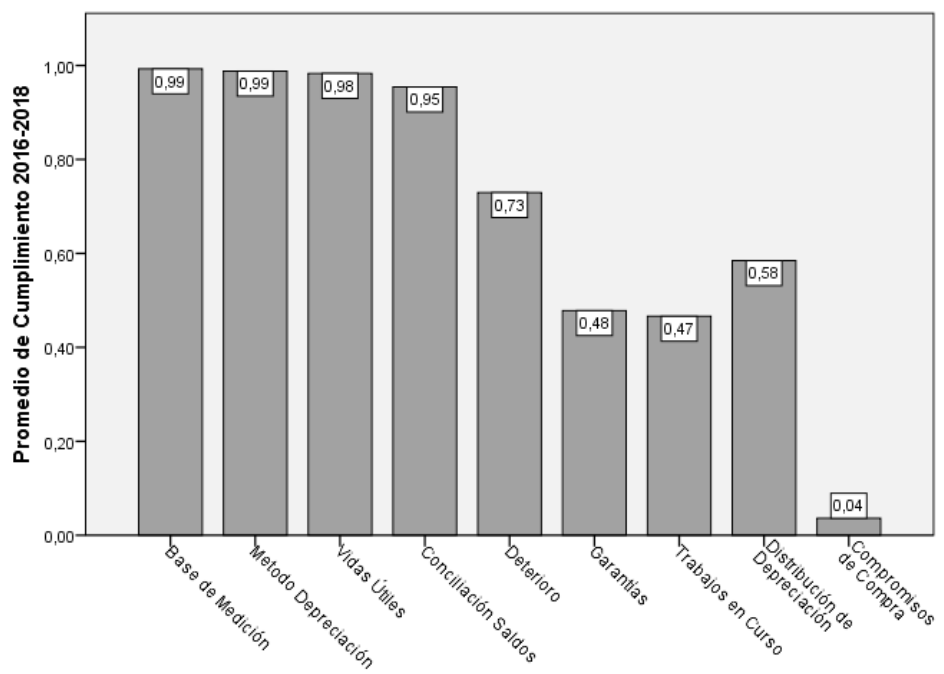

Fuente: elaboración propia.

La información sobre deterioro, restricción por garantías de activos, trabajos u obras en curso, distribución de la depreciación en las cuentas de resultados, y los compromisos de compras tienen un nivel de incidencia de revelación menor.

Respecto a la base de medición posterior de los activos, las empresas escogen como criterio de medición inicial el costo histórico. Las empresas, en un promedio del $14.49 \%$, para los tres años (figura 2) consideran el modelo de valor revaluado para sus PPE, aplicado principalmente para las siguientes clases de activos, terrenos y edificios, maquinarias y equipos en empresas agroindustriales, industriales, de energía y mineras. Estos resultados muestran que las empresas en el Perú usan en su mayoría un criterio 
conservador en la medición con el modelo de costo histórico de sus PPE y no de revaluación como una opción de la gerencia de mejorar la percepción de los inversores y acreedores sobre la situación financiera y capacidad de inversión (Jaggi y Tsui 2001; Hu et ál., 2015; Missonier-Piera, 2007) o por una ausencia de incentivos tributarios para elegirlo como práctica (Barlev et ál. 2007; Choi et ál., 2013).

Figura 2. Modelos de medición posterior de PPE

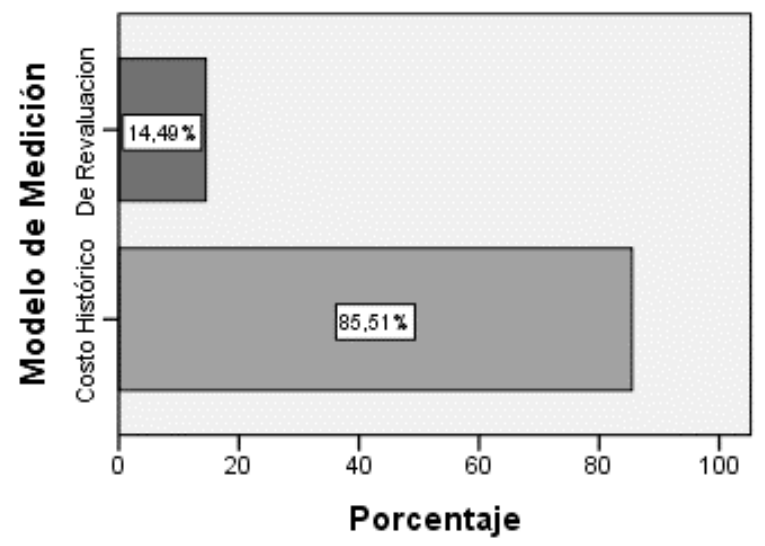

Fuente: elaboración propia.

Como se observa en la figura 3, el método de depreciación bajo línea recta es más usado por las empresas en un $95.65 \%$. Solo un $4.35 \%$ de las empresas aplica el método de unidades producidas, que es aplicado principalmente por las empresas agroindustriales en sus activos biológicos y plantas productoras, así como por las empresas mineras en sus maquinarias y equipos.

Estos hallazgos pueden ser considerados una limitación en la información, donde lo lógico sería que algunas empresas con actividad productiva o energía puedan aplicar modelos distintos al de línea recta y que se ajuste a la realidad empresarial de cada situación y a la intensión del IASB de presentar información de calidad que ayude a la toma de decisiones (Ahmed et ál., 2002; Pachón, 2010; Reeh y Molina, 2013). 
Figura 3. Métodos de depreciación

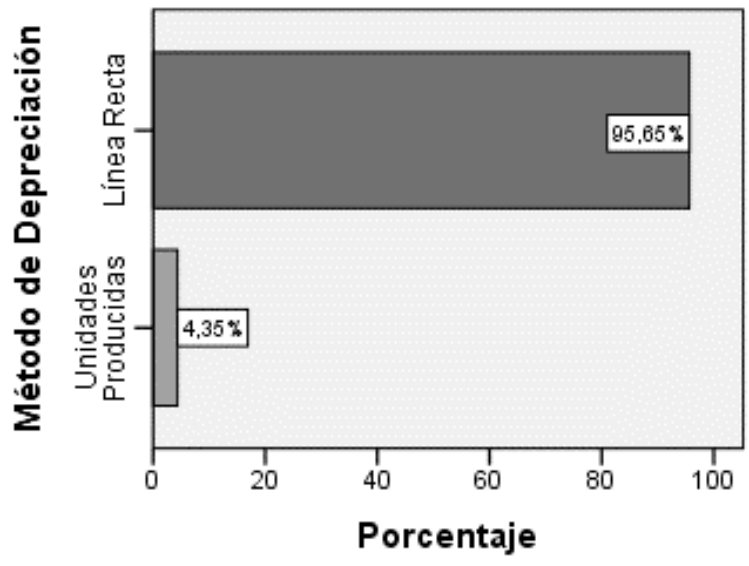

Fuente: elaboración propia.

Finalmente, en la figura 4 se muestra la media de cumplimiento de criterios de revelación de PPE de acuerdo con el sector empresarial para los tres periodos estudiados.

En primer lugar, el nivel de cumplimiento de criterios de revelación no varía significativamente a lo largo del periodo, pero si observamos diferentes niveles según la actividad de la empresa, destaca el nivel de revelación en los sectores de agroindustria, industria, minería y servicios públicos (empresas de energía, gas y telefonía) por ser empresas con importantes inversiones en edificaciones, terrenos, plantas, maquinarias, equipos para poder realizar su actividad industrial, producción y de servicio público, que inciden en el nivel de revelación en notas a los EEFF, caso distinto a empresas con menor inversión y uso de PPE como son las financieras, comerciales y de servicios (Ferreira da Costa y Morais de Oliveira, 2015; Hassan et ál., 2006; Kang y Gray, 2011; Stadler y Nobes, 2014). 
Figura 4. Nivel cumplimiento de revelación por sector y año

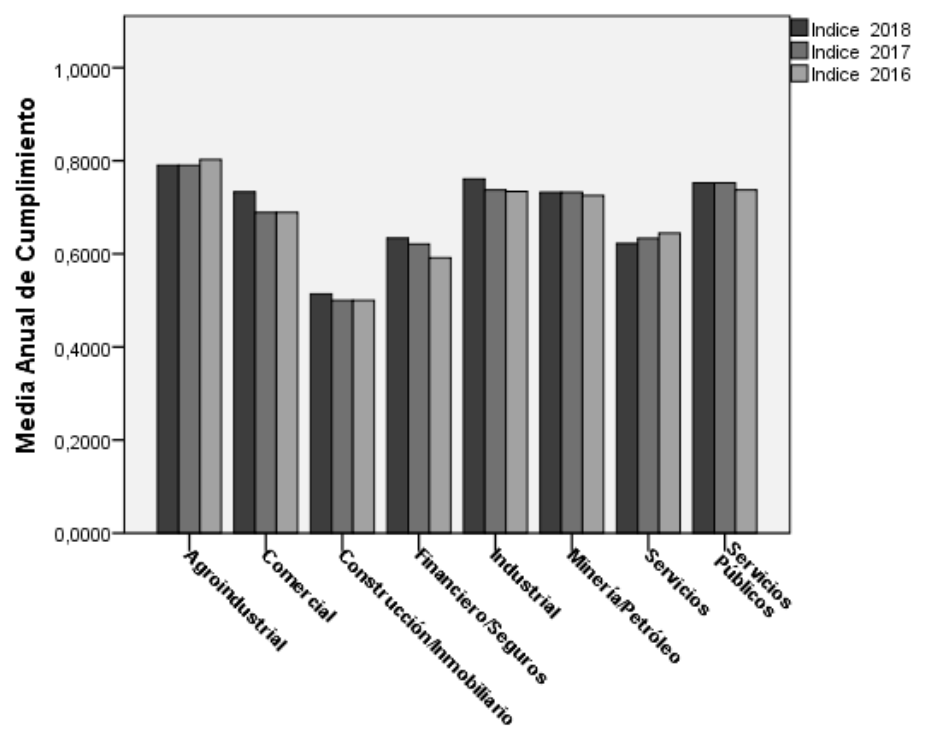

Fuente: elaboración propia.

\section{Análisis multivariante}

La tabla 3 resume los estadísticos descriptivos para todas las variables del modelo de regresión para las 138 empresas estudias para los tres años de estudio por un total de 414 observaciones.

El índice de cumplimiento de los indicadores solo llega a un promedio de 0.6911 sobre el máximo valor de 1 . Respecto a las variables continuas el tamaño promedio de las empresas estudiadas es 5000 millones de unidades monetarias de activos totales, con un nivel promedio de inversión en PPE de $36 \%$, antigüedad de 39 años y rentabilidad de $4.68 \%$ sobre el total de activos. 
Tabla 3. Estadísticos descriptivos

\begin{tabular}{|l|c|c|c|c|c|}
\hline \multicolumn{1}{|c|}{ Variable } & Media & $\begin{array}{c}\text { Desviación } \\
\text { estándar }\end{array}$ & Mín. & Máx. & N \\
\hline $\begin{array}{l}\text { Índice de } \\
\text { cumplimento }\end{array}$ & 0.6911 & 0.1476 & 0.0000 & 1.0000 & 414 \\
\hline Tamaño de activos & 4.9978 & 14.3680 & 0.0027 & 133.7656 & 414 \\
\hline Proporción PPE & 0.3593 & 0.3064 & 0.0001 & 0.9953 & 414 \\
\hline Antigüedad & 39.3768 & 27.2695 & 5.0000 & 141.0000 & 414 \\
\hline Firma de auditoría & 0.9058 & 0.2925 & 0.0000 & 1.0000 & 414 \\
\hline $\begin{array}{l}\text { Rentabilidad de } \\
\text { activos }\end{array}$ & 0.0468 & 0.0779 & -0.3223 & 0.4345 & 414 \\
\hline
\end{tabular}

Fuente: elaboración propia.

La tabla 4 de panel de regresión lineal muestra dos modelos, el primero (I) solo considera las variables independientes definidas para el estudio, el segundo modelo (II) considera además de las variables independientes los efectos de los años 2017 y 2018 respecto al 2016, en el nivel de cumplimiento.

Para controlar la correlación de las tres observaciones por cada empresa para los tres años consecutivos, se consideraron clústeres a nivel de firma observada tres veces, con ello los errores estándar resultan robustos y no presentan cambios importantes. Como se observó en el análisis descriptivo (figura 4), las empresas con importantes inversiones en PPE para poder realizar su actividad industrial, producción, minera y de servicio público (energía) presentan una mayor revelación de dichos activos (Hassan et ál., 2006; Kang y Gray, 2011; Lang y Stice-Lawrence, 2015; Stadler y Nobes, 2014).

En la tabla 4 se observa una influencia significativa de la variable sector en el nivel de divulgación por lo que no rechazamos la H1 $(p<0.01)$, resultando mayor la influencia para el grupo de empresas "industriales y energía" que el de "servicios y financieras". 
El tamaño de las empresas no resulta un factor determinante en la información revelada de PPE, por lo que rechazamos la hipótesis H2. La relación resulta negativa; los directivos de las grandes empresas estudiadas presentan menos incentivos de revelar una mayor información sobre sus PPE a los accionistas, determinados sectores como el financiero, construcción y de servicios de gran tamaño presentan un menor nivel de revelación debido a su menor inversión en PPE.

Estos resultados son contrarios a lo encontrado en la literatura (Ferreira da Costa y Morais de Oliveira, 2015; Istiningrum y Za'im Muhtadi, 2018; Uyar et ál., 2016).

La inversión en PPE medido por su proporción con respecto al total de activos se asocia positivamente y de forma significativa $(p<0.01)$ con el nivel de cumplimiento de revelación de dichos activos por lo que confirmamos la hipótesis $\mathrm{H} 3$.

Esto es consecuente con que las empresas de sectores específicos como el industrial, minería y energía estudiados (figura 4) con mayor inversión y uso de PPE presentan una mayor información revelada en notas a los EEFF (Ferreira da Costa y Morais de Oliveira, 2015; Kang y Gray, 2011; Stadler y Nobes, 2014).

Lo resultados muestran que la variable antigüedad de la empresa resulta un factor determinante de forma significativa $(p<0.05)$ en la revelación de información financiera, por lo que no rechazamos la Hipótesis H4. Las empresas que cuentan con más trayectoria en el mercado de valores de Lima y con más experiencia en preparar información presentan una mayor revelación sobre PPE como información para inversionistas y acreedores (Mutawaan y Hewaidy, 2010; Kang y Gray, 2011; Owusu-Ansah,1998). Encontramos que las empresas industriales, mineras y de energía tienen mayor inversión en PPE y antigüedad, por lo que muestran un mejor nivel de divulgación. 
Análisis del cumplimiento de revelación de propiedades, planta

y equipo para empresas que listan en la bolsa de valores de Perú

Tabla 4. Panel de regresión lineal

\begin{tabular}{|c|c|c|}
\hline \multicolumn{3}{|c|}{ Método de estimación: oLs } \\
\hline \multicolumn{3}{|c|}{ Variable dependiente: nivel de cumplimiento de revelación de PPE } \\
\hline & I & II \\
\hline \multirow{2}{*}{ Sector empresarial } & $0.07579^{* * *}$ & $0.07548^{* * *}$ \\
\hline & $(0.0267)$ & $(0.0267)$ \\
\hline \multirow{2}{*}{ Ln tamaño de activos } & -0.0018 & -0.0020 \\
\hline & $(0.0056)$ & $(0.0057)$ \\
\hline \multirow{2}{*}{ Inversión en PPE } & $0.1451^{* * *}$ & $0.1462^{* * *}$ \\
\hline & $(0.0423)$ & $(0.0424)$ \\
\hline \multirow{2}{*}{ Antigüedad de la empresa } & $0.0007^{\star *}$ & $0.0007^{\star *}$ \\
\hline & $(0.0003)$ & $(0.0003)$ \\
\hline \multirow{2}{*}{ Firma de auditoría } & $0.1472^{\star * *}$ & $0.1479^{* * *}$ \\
\hline & $(0.0327)$ & $(0.0330)$ \\
\hline \multirow{2}{*}{ Rentabilidad (ROA) año anterior } & 0.0178 & 0.0134 \\
\hline & $(0.0884)$ & $(0.0879)$ \\
\hline \multirow{2}{*}{ Año 2017} & - & 0.0121 \\
\hline & & $(0.0085)$ \\
\hline \multirow{2}{*}{ Año 2018} & - & $0.0226^{* *}$ \\
\hline & & $(0.0103)$ \\
\hline Observaciones & 414 & 414 \\
\hline R-cuadrado ajustado & 0.3899 & 0.3938 \\
\hline \multicolumn{3}{|l|}{ Error estándar en paréntesis } \\
\hline
\end{tabular}

Fuente: elaboración propia. 
La firma de auditoría es determinante en el nivel de divulgación de información sobre revelación de PPE en las notas a los EEFF; el tamaño de las firmas de auditoría se asocia significativamente con el nivel de revelación por lo que no rechazamos la hipótesis H5 $(p<0.01)$. Las firmas de auditoría pertenecientes al grupo de las "grandes" inciden en una mayor divulgación, esto se puede explicar debido a que estas sociedades buscan reducir el riesgo de auditoría y cuidar su reputación e incentivan a las empresas auditadas a que realicen un mayor cumplimiento normativo y regulatorio de revelación (Agyei-Mensah, 2019; Mutawaa y Hewaidy, 2010; García y Sánchez, 2006; Hodgdon et ál., 2009; Santos et ál., 2014).

No se encuentra que la rentabilidad sea un factor determinante en el nivel de revelación de PPE, por lo que rechazamos la hipótesis H6. La naturaleza del mercado de valores peruano se caracteriza por el financiamiento de las empresas principalmente con bancos y no con emisión de acciones. Esto puede implicar que las empresas más rentables no tengan incentivos de cumplir mayores requerimientos de revelación con accionistas (Ahmed y Courtis, 1999; Barako et ál., 2006; Uyar et ál., 2016).

Finamente se observa que en promedio el índice de revelación del 2018 y 2017 han aumentado y son mayores respecto al 2016.

\section{Conclusiones}

Esta investigación busca contribuir a los estudios existentes sobre el análisis de cumplimiento de los criterios de medición y factores de revelación de las PPE de acuerdo con los lineamientos de la NIC 16 en los EEFF de empresas cotizadas peruanas para los periodos 2016, 2017 y 2018.

En virtud de la importancia que tiene para las empresas y para los stakeholders presentar y recibir información financiera de calidad, trasparente y comparable sobre la información financiera para la oportuna toma de decisiones, se analizó el cumplimiento en la revelación de información 
financiera implementando la normativa vigente del IASB para sus propiedades, planta y equipo.

Como uno de los primeros hallazgos, podemos indicar que las empresas peruanas cotizadas muestran un alto nivel de revelación de las PPE en sectores con mayor inversión en PPE. La influencia del ente regulador del mercado de valores y las firmas de auditoría también resultan determinantes en este estudio (Ferreira da Costa y Morais de Oliveira, 2015; Pocomucha y Hernández-Pajares 2019).

A pesar de la mejora de calidad de información desde su primera aplicación hace más una década desde su primera adopción, observamos que para los periodos estudiados no se incrementa el nivel de revelación de forma importante. En la mayoría de los casos, la aplicación de criterios de medición sigue resultados conservadores y se elige como método de valoración posterior el modelo del costo histórico y métodos de depreciación lineales (Ahmed et ál., 2002; Reeh y Molina, 2013; Barlev et ál. 2007; Choi et ál., 2013).

Las inversiones en PPE y el tipo de industria al que pertenecen las empresas resulta factores determinantes en el nivel de divulgación es el caso de actividades empresariales como agroindustria, industria y servicios públicos con una significativa inversión en PPE, mayores recursos y menores costos de preparación de información (Stadler y Nobes, 2014; Tawiah y Boolaky, 2019). Siguiendo el mismo criterio, las entidades con mayor antigüedad en el mercado de valores y más experiencia en elaboración de información presentan un mayor cumplimento de los criterios de revelación (Mutawaa y Hewaidy, 2010; Owusu-Ansah,1998).

De acuerdo con la teoría de agencia, el mayor nivel de divulgación explicado por las variables de tamaño y rentabilidad no se ajusta a la realidad peruana. No tener un mercado de valores desarrollado explica por qué las empresas grandes no están interesadas en una mayor información a accionistas ni busquen reducir los costos de agencia (García y Sánchez, 2006; Kang y Gray, 2011). 
Sin embargo, la firma de auditoría si resulta determinante en el nivel de revelación, las cinco grandes firmas de auditoría en el Perú clasificadas como las "grandes", incentivan a las empresas que auditan al cumplimiento normativo para mejorar su reputación y cumplir con la calidad de trabajo de auditoría a comparación de las firmas de auditoría locales de menor tamaño (DeAngelo 1981; Santos et ál., 2014; Agyei-Mensah, 2019).

Esta investigación presentó limitaciones, pues el tamaño de la muestra es reducido por lo que no se podrán extrapolar los resultados. Se sugiere que para estudios futuros se realicen análisis comparativos con empresas de otros países de la región con naturaleza económica similar. Por otro lado, se deben considerar otras variables que resulten factores determinantes en futuras investigaciones como el valor de capitalización bursátil o internacionalización.

\section{Referencias}

Agyei-Mensah, B. K. (2019). IAS-38 disclosure compliance and corporate governance: evidence from an emerging market. Corporate Governance: The International Journal of Business in Society, 19(3), 419-437.

Ahmed, K., y Courtis, J. K. (1999). Associations between corporate characteristics and disclosure levels in annual reports: a meta-analysis. The British Accounting Review, 31(1), 35-61.

Ahmed, A., Billings, B. K., Morton, R. M. y Stanford-Harris, M. (2002). The role of accounting conservatism in mitigating bondholder-shareholder conflicts over dividend policy and in reducing debt costs. The Accounting Review, 77(4), 867-890.

Alanezi, F. S., Alfraih, M. M. y Alshammari, S. S. (2016). Operating Segments (IFRS 8)-Required Disclosure and the Specific-Characteristics of Kuwaiti Listed Companies. International Business Research, 9(1), 136.

Alanezi, F. S. y Albuloushi, S. S. (2011). Does the existence of voluntary audit committees really affect IFRs-required disclosure? The Kuwaiti evidence. International Journal of Disclosure and Governance, 8(2), 148-173. 
Aljifri, K. y Khasharmeh, H. (2006). An investigation into the suitability of the international accounting standards to the United Arab Emirates environment. International Business Review, 15(5), 505-526.

Barako, D. G., Hancock, P. y Izan, H. Y. (2006). Factors influencing voluntary corporate disclosure by Kenyan companies. Corporate Governance: an international review, 14(2), 107-125.

Barlev, B., Fried, D., Haddad, J. y Livnat, J. (2007). Reevaluation of revaluations: a cross-country examination of the motives and effects on future performance. Journal of Business Finance \& Accounting, 34, 1025-1050.

Birt, J. L., Bilson, C. M., Smith, T. y Whaley, R. E. (2006). Ownership, competition, and financial disclosure. Australian Journal of Management, 31(2), 235-263.

Callao, S., Jarne, J. I., y Laínez, J. A. (2007). Adoption of IFRs in Spain: Effect on the comparability and relevance of financial reporting. Journal of International Accounting, Auditing and Taxation, 16(2), 148-178.

Cano, A. M. (2010). Algunas experiencias exitosas en Latinoamérica relacionadas con la estandarización contable. Revista Facultad de Ciencias Económicas, 18(2), 207-226.

Cardona, J. C. (2018). Impacto de las IFRS en la calidad del reporte financiero en Latinoamérica y el Caribe. Contaduría y Administración, 63 (Especial. 2), 841-861.

Cascino, S., y Gassen, J. (2015). What drives the comparability effect of mandatory IFRS adoption? Review of Accounting Studies, 20(1), 242-282.

Católico-Segura, D., Pulido-Ladino, J., y Cely-Angarita, V. (2013). Revelación de la información financiera sobre propiedad, planta y equipo en empresas industriales cotizadas en Colombia. Cuadernos de Contabilidad, 14(36), 943-970.

Choi, F. D. y Meek, G. K. (2008). International accounting. Pearson Education.

Choi, T. H., Pae, J., Park, S. y Song, Y. (2013). Asset revaluations: motives and choice of items to revalue. Asia-Pacific Journal of Accounting \& Economics, 20(2), 144-171.

Cooke, T. E. (1992). The impact of size, stock market listing and industry type on disclosure in the annual reports of Japanese listed corporations. Accounting and Business Research, 22(87), 229-237. 
Crowe Howarth. (2017). World survey: what an uncertain world. International Accounting Bulletin n. ${ }^{\circ}$ 570. Consultado en http://www.crowehorwath.ie/ wp-content/uploads/2017/02/IAB-2017_World-Survey.pdf

Daske, H., Hail, L., Leuz, C. y Verdi, R. (2013). Adopting a label: Heterogeneity in the economic consequences around IAS/IFRS adoptions. Journal of Accounting Research, 51(3), 495-547.

DeAngelo, L. E. (1981). Auditor size and audit quality. Journal of Accounting and Economics, 3(3), 183-199.

De Moura, A. A. y Gupta, J. (2019). Mandatory adoption of IFRs in Latin America: A boon or a bias. Journal of International Financial Markets, Institutions and Money, 60, 111-133.

Díaz-Becerra, O. A. (2010). Estado actual de la aplicación de las NIIF en la preparación de estados financieros de las empresas peruanas. Contabilidad $y$ Negocios, 5(10), 5-28.

Díaz-Becerra, O. A., Rojo, L. A. y Medina, A. V. (2012). Análisis de las diferencias entre el tratamiento contable y el fiscal para los elementos de propiedades, planta y equipo: el caso peruano. Contabilidad yNegocios, 7(14), 5-22.

Erdem, S., Aslanertik, E. y Yardimci, B. (2017). The main determinants of differences in compliance levels of disclosure items for IAS 16 in BIST. Journal of Financial Reporting and Accounting, 15(3), 317-332.

Easton, P. D., Eddey, P. H. y Harris, T. S. (1993). An investigation of revaluations of tangible long-lived assets. Journal of Accounting Research, 31, 1-38.

Ferreira da Costa, F. y Morais de Oliveira, L. C. (2015). A divulgação dos ativos fixos tangíveis segundo a IAs 16 eo seu grau de cumprimento. Innovar, 25(spe), 47-60.

García, E., y Sánchez, J. P. (2006). Un estudio meta-analítico de los factores determinantes de la revelación de información. Spanish Journal of Finance and Accounting, 35(132), 761-788.

Giner, B, Cervera, N., Ruiz, A., y Arce, M. (2003). Incentivos para la divulgación voluntaria de información: evidencia empírica sobre la información segmentada. Revista Europea de Dirección y Economía de la Empresa, 12(4), 69-85.

Jaggi, B., y Tsui, J. (2001). Management motivation and market assessment: revaluations of fixed assets. Journal of International Financial Management and Accounting, 12(2), 160-187. 
Jara, L., Contreras, H. y Castro, B. (2010). Adopción de IfRS en empresas chilenas del sector eléctrico que cotizan en bolsa. Revista Internacional Legis de Contabilidad y Auditoría, 42, 131-163.

Johnston, K. (2014). Organic vs. Inorganic Growth in Business. Consultado en https:// smallbusiness.chron.com/organic-vs-inorganic-growth-business-37311.html

Hassan, O., Giorgioni, G. y Romilly, P. (2006), The extent of accounting disclosure and its determinants in an emerging capital market: the case of Egypt. International Journal of Accounting, Auditing and Performance Evaluation, 3(1), 41-67.

Hellman, N., Carenys, J. y Moya Gutierrez, S. (2018). Introducing more IFRs principles of disclosure-will the poor disclosers improve? Accounting in Europe, 15(2), 242-321.

Hernández, J., Núñez, I. y Zapata, D. (2017). Criterios de medición y revelación de la NIC 41 aplicados por empresas peruanas y chilenas. Teuken Bidikay, 8(10), 118-132.

Hodgdon, C., Tondkar, R. H., Adhikari, A. y Harless, D. W. (2009). Compliance with International Financial Reporting Standards and auditor choice: New evidence on the importance of the statutory audit. The International Journal of Accounting, 44(1), 33-55.

Horton, J., Serafeim, G. y Serafeim, I. (2013). Does mandatory IFRS adoption improve the information environment? Contemporary accounting research, 30(1), 388-423.

Hu, F., Percy, M. y Yao, D. (2015). Asset revaluations and earnings management: Evidence from Australian companies. Corporate Ownership and Control, 13(1), 930-939.

Istiningrum, A. A. y Zảim Muhtadi, M. Z. (2018). The Determinants and Impact of Mandatory Disclosure in Indonesian Manufacturing Companies. Journal of Economics, Business \& Accountancy Ventura, 21(1), 25-39.

Kang, H. H. y Gray, S. J. (2011). Reporting intangible assets: Voluntary disclosure practices of top emerging market companies. The International Journal of Accounting, 46(4), 402-423.

Lang, M. y Lundholm, R. (1993). Cross-sectional determinants of analyst ratings of corporate disclosures. Journal of Accounting Research, 31(2), 246-271. 
Lang, M. y Stice-Lawrence, L. (2015). Textual analysis and international financial reporting: Large sample evidence. Journal of Accounting and Economics, 60(2-3), 110-135.

Meek, G. K. y Gray, S. J. (1989). Globalization of stock markets and foreign listing requirements: Voluntary disclosures by continental European companies listed on the London Stock Exchange. Journal of International Business Studies, 20(2), 315-336.

Miihkinen, A. (2012). What drives quality of firm risk disclosure?: the impact of a national disclosure standard and reporting incentives under IFRs. The International Journal of Accounting, 47(4), 437-468.

Missonier-Piera, F. (2007). Motives for fixed-asset revaluation: An empirical analysis with Swiss data. The International Journal of Accounting, 42(2), 186-205.

Molina-Llopis, R. M., Díaz-Becerra, O. D., Capuñay, J. C. y Casinelli, H. (2014). El proceso de convergencia con las Normas Internacionales de Información Financiera en España, Perú y Argentina. Contabilidad y Negocios, 9(18), 6-26.

Mongrut, S. y Winkelried, D. (2019). Unintended effects of IFRS adoption on earnings management: The case of Latin America. Emerging Markets Review, 38, 377-388.

Mutawaa, A. and Hewaidy, A, M. (2010), Disclosure Level and Compliance with IFRSS: An Empirical Investigation of Kuwaiti Companies. International Business and Economics Research Journal, 9(5). Dor: https://doi.org/10.19030/iber. v9i5.566

Owusu-Ansah, S. (1998). The impact of corporate attribites on the extent of mandatory disclosure and reporting by listed companies in Zimbabwe. The International Journal of Accounting, 33(5), 605-631.

Pachón, M. A. (2010). La aplicación de criterios prudentes en la valoración de activos, costos, gastos y pasivos. Activos, 8(14), 159-169.

Paik, G. (2009). The value relevance of fixed asset revaluation reserves in international accounting. International Management Review, 5(2), 73.

Pocomucha K. y Hernández-Pajares J. (2019) Influencia de la firma de auditoría y desempeño de empresas cotizadas en la extensión de revelación en reportes anuales. Contabilidad \& Sistemas, 16, 46-54. 
Reeh, M. M. y Molina, N. A. (2013). La crisis del concepto prudencia a causa del tránsito de las normas nacionales austriacas de información financiera a las normas internacionales. Contaduría y Administración, 58(2), 91-112.

Salas, J., Vilchez, P. y Curvelo, J. (2017). Claridad y calidad de las notas explicativas en estados financieros bajo IFRs. Un estudio de brechas de Expectativas Gaps para normas contables (NIC 2, NIC 16, NIC 37 y NIC 38). Quipukamayoc, 25 (49), 27-40.

Samaha, K., Khlif, H. y Dahawy, K. (2016). Compliance with IAS/IFRS and its determinants: a meta-analysis. Journal of Accounting, Business and Management, 23(1), 41-63.

Santos, E., Ponte, V. y Mapurunga, P. (2014). Mandatory IFRS adoption in Brazil (2010): Index of compliance with disclosure requirements and some explanatory factors of firms reporting. Revista Contabilidade \& Finanças, 25(65), 161-176.

Silva, B. y Garrido, C. (2006). La convergencia a las normas internacionales de información financiera en Chile. Capic Review, (4), 9-19.

Stadler, C., y Nobes, C. W. (2014). The influence of country, industry, and topic factors on IFRs policy choice. Abacus, 50(4), 386-421.

Superintendencia del Mercado de Valores (SMV). Ley $N^{\circ} 29720$. Consultado en https://www.smv.gob.pe/Frm_SIL_Detalle.aspx?CNORMA=LEY000020112 9720\&CTEXTO $=$

Superintendencia del Mercado de Valores (SMV). Resolución $N^{\circ}$ 011-2012. Smv/01. Consultado en https://www.smv.gob.pe/Frm_SIL_Detalle. aspx?CNORMA $=$ RSA 00001600014\% \%20\% \%20\&CTEXTO=

Uyar, A., Kılıç, M. y Gökçen, B. A. (2016). Compliance with IAs/IFRS and firm characteristics: evidence from the emerging capital market of Turkey. Economic research-Ekonomska istraživanja, 29(1), 148-161.

Tawiah, V. y Boolaky P. (2019). Determinants of IfRS compliance in Africa:analysis of Stakeholder Attributes. International Journal of Accounting \& Information Management, 27(4), 573-599.

Yáñez-Andrades, V.; Pilar-Cortés, F. e Inostrosa-Gonzáles, C. (2010). Impacto de las IFRS en la valoración de empresas: evidencia para una muestra de empresas chilenas cotizadas. Revista Activos, 15, 153-182.

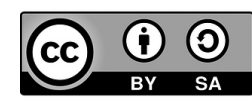

